\title{
Reduced risk of next exacerbation and mortality associated with antibiotic use in COPD
}

\author{
B.M. Roede*,\#, P. Bresser", J.M. Prins*, F. Schellevis ${ }^{+, \S}$, \\ T.J.M. Verheij ${ }^{f}$ and P.J.E. Bindels ${ }^{\#}$
}

ABSTRACT: The long-term risk of a subsequent exacerbation of chronic obstructive pulmonary disease (COPD) after treatment with oral corticosteroids without (OS) or with antibiotics (OSA) was compared in a historical general practice-based cohort.

Eligible patients were $\geqslant 50 \mathrm{yrs}$ of age, had a registered diagnosis of COPD, were on maintenance respiratory drugs, and had experienced at least one exacerbation defined as a prescription OS or OSA. Times to second and third exacerbations were assessed using KaplanMeier survival analysis; the risk of a subsequent exacerbation was assessed in a Cox proportional hazards analysis; and all-cause mortality was assessed using Kaplan-Meier survival and Cox proportional hazards analyses.

A total of 842 patients had one or more exacerbations. The median time from first to second exacerbation was comparable for the OS and OSA groups, but the time from second to third exacerbation differed: 189 versus 258 days, respectively. The protective effect of OSA was most pronounced during the first 3 months following treatment (hazards ratio $0.72,95 \%$ confidence interval 0.62-0.83). Exposure to antibiotics unrelated to a course of oral corticosteroids almost halved the risk of a new exacerbation. Mortality during follow-up was considerably lower in the OSA group.

Adding antibiotics to oral corticosteroids was associated with: reduced risk of subsequent exacerbation, particularly in patients with recurrent exacerbations; and reduced risk of all-cause mortality.

KEYWORDS: Antibiotics, chronic obstructive pulmonary disease, exacerbation, oral corticosteroids, primary healthcare

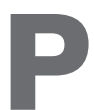

atients with an acute exacerbation of chronic obstructive pulmonary disease (COPD) are often treated with a combination of antibiotics and corticosteroids. However, only patients with severe symptoms, and/or patients with a low baseline expiratory flow rate appear to benefit from antibiotic treatment [1-3]. A recent Cochrane Review article supports the use of antibiotics for exacerbations of COPD with increased cough and sputum purulence in patients who are moderately or severely ill [4]. Analysis restricted to community-based studies, however, did not demonstrate a difference between antibiotic and placebo [4]. In addition, most studies on the use of antibiotics in exacerbations of COPD have focused on the evaluation of short-term recovery from exacerbation, with a follow-up period of only a few weeks.

In a previous population-based cohort study by the present study group [5] that used pharmacy dispensing records [6], it was demonstrated that treatment of an exacerbation with antibiotics in addition to oral corticosteroids was associated with an increased time to a subsequent exacerbation and an improved survival. Extrapolation of those results to patients diagnosed with COPD, however, must be done with caution; the authors studied a heterogeneous population defined by the use of respiratory drugs for the maintenance treatment of obstructive lung disease [5].

Therefore, the present authors' aim in the current study was to extend the observations to a welldefined COPD population using the Second Dutch National Survey of General Practice (DNSGP-2) [7].

\section{METHODS}

\section{Data sources}

The data used in the present study were derived from: the DNSGP-2, carried out by the

\section{AFFILIATIONS}

*Dept of Internal Medicine, Division of Infectious Diseases, Tropical Medicine and AIDS, and Center for Infection and Immunity Amsterdam (CINIMA),

Depts of \#General Practice and

"Pulmonology, Academic Medical

Centre, University of Amsterdam, and

${ }^{+}$Dept of General Practice/EMGO

Institute VU University Medical

Centre, Amsterdam,

${ }^{\S}$ Netherlands Institute for Health Services Research (NIVEL), and fJulius Center for Health Sciences and Primary Care, University Medical Center, Utrecht, The Netherlands.

CORRESPONDENCE

B.M. Roede

Academic Medical Centre

University of Amsterdam

Dept of Internal Medicine

Division of Infectious Diseases

Tropical Medicine

and AIDS

Room F4-217

Meibergdreef 9

1105 AZ Amsterdam

The Netherlands

Fax: 31206972286

E-mail: ineke.roede@rivm.nl

Received:

June 112008

Accepted after revision:

October 032008

STATEMENT OF INTEREST

None declared. 
Netherlands Institute for Health Services Research (NIVEL) in 2001 [7]; and the National Information Network of General Practice (LINH) [8]. DNSGP-2 was performed in 104 general practices in the Netherlands, comprising 195 general practitioners (GPs) and including 400,911 patients; it provides a representative impression of the morbidity and prescribing habits in Dutch general practice [7]. The patients in these practices are representative of the Dutch general population with respect to age, sex and type of healthcare insurance. LINH, connected with NIVEL, is a computerised network of 85 general practices with almost 340,000 registered patients, providing representative information with respect to care delivered by Dutch GPs, based on data from electronic medical records. In the DNSGP-2 data were collected for $1 \mathrm{yr}$; LINH collects data continuously. General practices were selected when participating both in the DNSGP-2 as well as in the LINH registration network.

\section{Patient selection}

The practices selected from the DNSGP-2 and LINH databases were those practices which provided complete data on morbidity and prescriptions from the cohort entry date, the date of the general practice entering the DNSGP-2 in 2001, until 31 December 2005. Patients included were those who: in the first year of participating in the DNSGP-2 were registered with COPD, according to International Classification of Primary Care code (ICPC) R95 [9]; and, in addition, in the first year of participating in the DNSGP-2 had at least two prescriptions of maintenance respiratory drugs, coded Anatomical Therapeutic Chemical (ATC) R03 [10]. These prescriptions included inhaled $\beta_{2}$-agonists, inhaled anticholinergics, inhaled corticosteroids, oral theophylline, or a combination of these agents. In order to minimise the number of patients with asthma, only those aged $\geqslant 50$ yrs were included. For the same reason, patients using leukotriene receptor antagonists (LTRAs; montelukast, ATC code R03DC03) or cromoglycates (R03BC) were also excluded.

Patient characteristics (age, sex, maintenance medication for obstructive lung disease, co-medication for cardiovascular disease and diabetes mellitus) were derived from the DNSGP-2 database.

\section{Definition of exacerbation}

COPD patients were selected who had experienced one or more exacerbation during the follow-up period. An exacerbation was defined as "a prescription of a short course of oral corticosteroids, with or without antibiotics". The date of prescription of oral corticosteroids (ATC code: H02AB06/ H02AB07), with or without antibiotics, was recorded. The following antibiotics were selected: doxycyclin (ATC-code: J01AA02); amoxicillin (J01CA04); amoxicillin-clavulanate (J01CR02); erythromycin (J01FA01); azithromycin (J01FA10); clarithromycin (J01FA09); ciprofloxacin (J01MA02); moxifloxacin (J01MA14); and levofloxacin (J01MA12). In the Netherlands, these antibiotics cover almost all antibiotics prescribed for exacerbations of COPD in primary care [11]. Episodes treated with antibiotics only were not included, as diagnoses were only available in some of the patients and therefore these prescriptions could not automatically be considered to represent an exacerbation.
When more than one course of oral corticosteroids was prescribed within a period of 3 weeks, this episode was considered as a single exacerbation. If the time between two prescriptions exceeded 3 weeks, this second episode was considered as a new exacerbation, as in the majority of patients symptoms are usually considerably improved 3 weeks after the onset of an exacerbation [12].

Patients prescribed oral corticosteroids or antibiotics for $>21$ days at regular intervals, for a period of 3 months or longer, were excluded from the analysis, since these patients cannot be discriminated from patients on maintenance treatment.

\section{Statistical analysis}

Treatment groups were compared at time of first exacerbation with respect to age, sex, use of inhaled respiratory drugs, including inhaled corticosteroids, and co-medication for cardiovascular disease or diabetes.

The first exacerbation after cohort entry was assessed and the time to the second exacerbation was calculated. The date of prescribing the exacerbation medication was considered to be the start of the second exacerbation. The time between the second and the third exacerbation was also calculated. These time periods were compared between patients treated with oral corticosteroids only (OS) and those treated with oral corticosteroids and antibiotics (OSA) using a Kaplan-Meier survival analysis. Patients were censored for exacerbation-free survival.

The effect of the variable of primary interest, treatment of exacerbation (i.e. OS (coded as 0) or OSA (coded as 1)), was analysed in a Cox proportional hazards model. All exacerbations from each patient were used, and the time was set back to zero after each exacerbation (gap-time unrestricted model) [13]. Hence, each exacerbation was treated as a separate record and the time since last exacerbation was treated as the principal timescale. A correction for recurrent exacerbation events from the same individual was made by including a frailty term in the model [14]. The Schoenfeld residuals as obtained from the model of time to next exacerbation suggested the difference in treatment effect to be highly nonproportional. Therefore, the difference in treatment effect was allowed to change at 3 months, 6 months and 1 yr. The data were coded so that hazard ratios (HRs) below unity indicated a preventive effect of adding an antibiotic to the oral corticosteroids. Potential confounding by the following patient characteristics was controlled for: age, sex, number of prescriptions of respiratory drugs, including inhaled corticosteroids, and co-medication for cardiovascular disease (yes/no) or for diabetes (yes/no) [15]. Prescriptions of antibiotics without oral corticosteroids were also treated as a time-dependent covariate, and assumed to be of influence for a period of 3 months; 3 months after this antibiotic was prescribed, the variable was again coded as "no antibiotic".

All-cause mortality of both treatment groups during follow-up was analysed using Kaplan-Meier and Cox proportional hazards analyses, and 95\% confidence intervals (CIs) were calculated.

\section{RESULTS}

\section{Patients}

From the DNSGP-2/LINH database, 54 practices, comprising 216,455 patients, provided complete data on morbidity and 


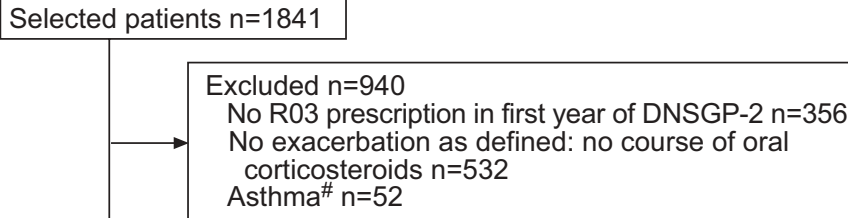

Patients with one or more exacerbation $\llbracket n=901$

Oral corticosteriods not for COPD $n=30$

Oral corticosteriods as maintenance treatment for COPD $n=10$

Antibiotics as maintenance treatment $n=13$

No "at risk" time $n=6$

In analysis $\mathrm{n}=842$

FIGURE 1. Flow diagram of patients in the analysis. \#: asthma medication such as leukotriene receptor antagonists or cromones; ": prescription oral corticosteroids with or without antibiotics. R03: maintenance respiratory drugs, coded Anatomical Therapeutic Chemical R03; DNSGP-2: the Second Dutch National Survey of General Practice; COPD: chronic obstructive pulmonary disease.

prescriptions in the selected time period. Of these patients, 63,721 were aged $>50 \mathrm{yrs}$, and 1,841 were identified as patients diagnosed with COPD (2.9\%). Of the 1,841 patients, 999 were excluded. A total of 356 did not meet the pre-specified criteria on the use of maintenance respiratory drugs; 532 patients did not experience a single exacerbation, as they were never prescribed oral corticosteroids; and 52 patients used LTRAs or cromoglycates. Out of the 1,841 patients originally identified, this left 901 patients with one or more exacerbation. Of these: 30 were excluded because the oral corticosteroids were prescribed for indications other than COPD; 10 were on maintenance treatment with oral corticosteroids for COPD; 13 were on maintenance treatment with antibiotics; and six were not "at risk" for a following exacerbation (only one exacerbation $<21$ days before the end of follow-up). This left 842 patients for the final analysis (fig. 1).

Of these 842 patients, 144 (17\%) died during the follow-up period. The cause of death in 23 patients was reported not to be related to COPD. A total of 13 patients died as a result of COPD; this was counted as an event and not right-censored. In the remaining 108 patients who died during follow-up, the cause of death could not be retrieved from the database. The median follow-up time after the first exacerbation was 1,353 (interquartile range (IQR) 791-1,649) days (or 3 yrs and 8.5 months). In total, 842 patients were followed for 2,723 person-yrs in total.

Of 842 patients having had at least one predefined exacerbation, 404 were treated at first exacerbation with OS, and 438 were treated with OSA. These groups were similar with respect to age, sex, respiratory medication, and use of comedication for diabetes and cardiovascular disease (table 1).

Of all 4,038 exacerbations (median 3 (IQR 1-6) per patient), GPs prescribed OS in 54\% and OSA in $46 \%$. Antibiotics used in the treatment of first exacerbations were doxycyclin $(n=218)$ penicillins (amoxicillin-clavulanate and amoxicillin, $n=141$ ), macrolides (azithromycin, clarithromycin and erythromycin, $\mathrm{n}=68$ ) and fluoroquinolones (ciprofloxacin, moxifloxacin and levofloxacin, $\mathrm{n}=11$ ).

\section{Kaplan-Meier estimates of developing a second exacerbation according to treatment type (OS or OSA)}

Overall, 595 patients (71\%) experienced a second exacerbation. The median time to the second exacerbation was 331 (95\% CI 258-404) days in the OS group compared to 312 (95\% CI 265359 ) days in the OSA group ( $p=0.31$; fig. $2 a)$. At 6 month after the first exacerbation, $27 \%$ of patients treated with OS had experienced a second exacerbation compared to $23 \%$ of patients treated with OSA; after $1 \mathrm{yr}$ this was $52 \%$ and $54 \%$, respectively.

\section{Kaplan-Meier estimates of developing a third exacerbation according to treatment type (OS or OSA)}

Of the 595 patients who experienced a second exacerbation, $308(52 \%)$ were treated with OS and $287(48 \%)$ were treated with OSA. A total of 450 patients experienced a third exacerbation during follow-up. The time between the second and the third exacerbation was much shorter in the OS group than in the OSA group: median time 189 (95\% CI 149-229) days compared to 258 (95\% CI 198-318) days, respectively $(\mathrm{p}<0.01$ fig. $2 b$ ). At 6 months after the second exacerbation, $50 \%$ of patients treated with OS had experienced a third exacerbation, compared to $41 \%$ of patients treated with OSA; after $1 \mathrm{yr}$ this was $69 \%$ and $61 \%$, respectively.

\section{Cox analysis of developing a new exacerbation according to treatment type}

In a univariate Cox regression model, the HR of an exacerbation after treatment with OSA compared to OS was 0.73 (95\% CI 0.63-0.84). In a Cox proportional hazards model, adjusting for potential confounding factors, the $\mathrm{HR}$ of an exacerbation after treatment with OSA was 0.72 (95\% CI 0.62-0.83) in the first 3 months following treatment, but the effect was not significant in subsequent time periods. In addition, the use of antibiotics prescribed without a course of oral corticosteroids,

\begin{tabular}{|c|c|c|c|}
\hline \multirow[t]{2}{*}{ TABLE 1} & \multirow[b]{2}{*}{ Treated with os } & $\begin{array}{l}\text { according to } \\
\text { tion }\end{array}$ & \multirow[b]{2}{*}{ p-value } \\
\hline & & OSA & \\
\hline Subjects n & 404 & 438 & \\
\hline Age yrs & $70(62-78)$ & $71(63-77)$ & 0.30 \\
\hline Males & $217(54)$ & 231 (53) & 0.78 \\
\hline Respiratory prescriptions & $8(4-12)$ & $8(4-13)$ & 0.35 \\
\hline Using ICS & $313(77)$ & $360(82)$ & 0.88 \\
\hline \multicolumn{4}{|l|}{ Co-medication use } \\
\hline Cardiovascular & $301(74)$ & $323(74)$ & 0.80 \\
\hline Diabetes & $53(13)$ & $50(11)$ & 0.45 \\
\hline Follow-up time days & $1322(750-1627)$ & $1375(832-1657)$ & \\
\hline \multicolumn{4}{|c|}{ 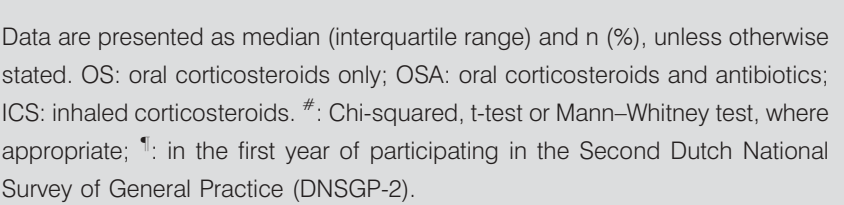 } \\
\hline
\end{tabular}



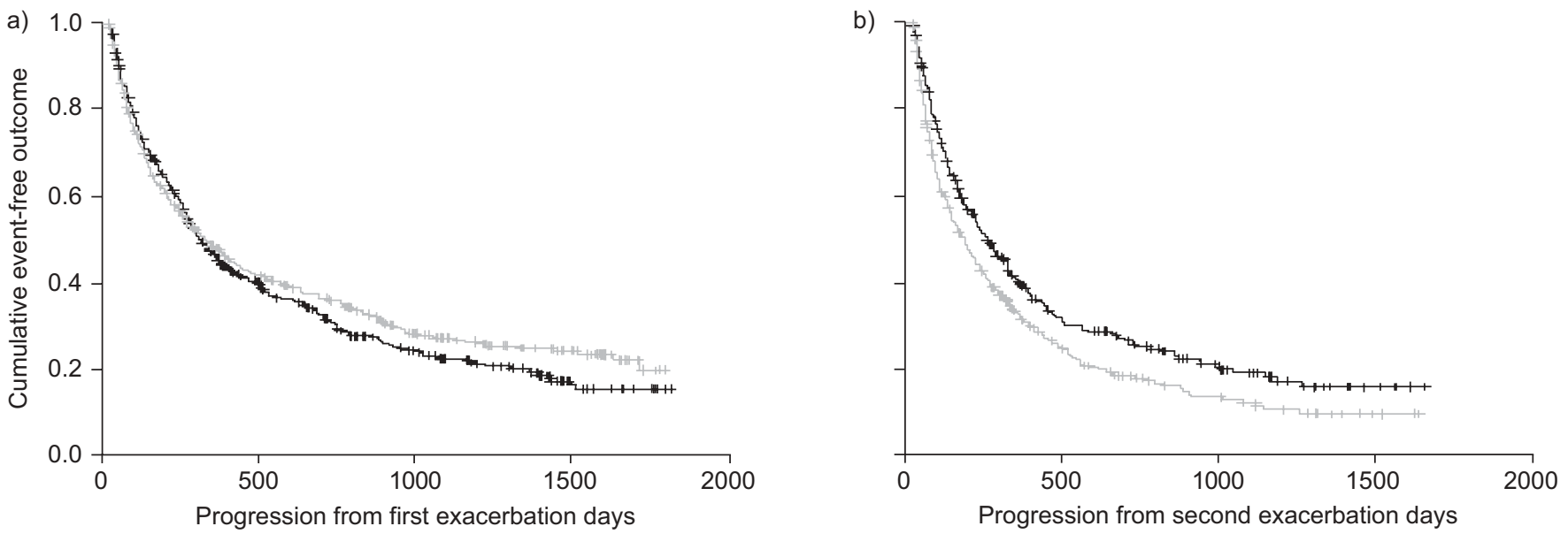

FIGURE 2. Kaplan-Meier estimates of the fraction of patients free of a) a second or b) a third exacerbation, stratified according to treatment type (grey line: oral corticosteroids only; black line: oral corticosteroids and antibiotics). Calculated median difference between both treatment groups is 19 days, $p=0.31$, by log-rank (a). Calculated median difference between both treatment groups is 69 days, $p<0.01$, by log-rank (b). a) $n=842 ; b) n=595$.

irrespective of the indication, almost halved the risk of a subsequent exacerbation (HR 0.56, 95\% CI 0.48-0.71; table 2).

\section{Mortality of both treatment groups during follow-up}

After the first exacerbation, during follow-up $62(14 \%)$ of the 438 patients died in the OSA group compared to $82(20 \%)$ of the 404 in the OS group ( $\mathrm{p}=0.02$; fig. 3 ). The HR of all-cause mortality after treatment with oral corticosteroids and antibiotics compared to corticosteroids only was 0.67 (95\% CI 0.48-0.93) in a univariate Cox regression model. In a Cox proportional hazards model, adjusting for the potential confounders, the HR was 0.62 (95\% CI 0.45-0.87).

\section{DISCUSSION}

The long-term risk of a subsequent exacerbation after treatment with OSA or OS was compared in COPD patients treated by GPs and using maintenance respiratory medication. The results showed that treatment of exacerbations with OSA and also the use of antibiotics without oral corticosteroids is associated with a reduced risk of a subsequent exacerbation. Moreover, a survival benefit was shown in the OSA group. To the present authors' knowledge, the demonstrated effect of antibiotic treatment on the risk of a subsequent exacerbation as well as the associated survival benefit has not previously been reported in an extensive population-based COPD cohort.

\section{Strengths and limitations of the present study}

The DNSGP-2/LINH database provided a selection of COPD patients, defined according to ICPC code R95 [9]. One limitation of using a registration database was that available data were not collected for the specific aim of the present study; therefore, relevant information, such as lung function data, may be lacking. In addition, no clinical information on the severity of the exacerbation was present, which may have caused treatment selection bias. However, relevant baseline characteristics of the treatment groups were similar. Furthermore, patients treated with OSA are likely to have more severe exacerbations compared to patients treated with

\section{TABLE 2 Hazard ratios (HRs) of developing a subsequent exacerbation, in a Cox proportional hazards model}

\section{HR $(95 \% \mathrm{Cl})$ of subsequent exacerbation}

\section{OSA versus OS \\ $0-3$ months after treatment \\ 3-6 months after treatment \\ 6-12 months after treatment \\ $>12$ months after treatment}

Exposure to antibiotics after previous exacerbation

Female sex

Inhaled corticosteroids as maintenance medication ${ }^{\#}$

Co-medication

Cardiovascular ${ }^{\#}$

Diabetes $^{\#}$
$0.72(0.62-0.83)$
$0.85(0.70-1.04)$
$1.02(0.80-1.30)$
$1.22(0.89-1.66)$
$0.56(0.45-0.71)$
$0.91(0.75-1.10)$
$0.87(0.68-1.12)$
$1.37(1.10-1.73)$
$0.90(0.68-1.19)$

The variables "age" and "number of respiratory drug prescriptions" were included in the Cox model but were not fitted linearly; therefore, their HRs are not presented. The risk of a new exacerbation increased significantly with age and with a higher number of prescriptions of respiratory drugs. OSA: antibiotics and oral corticosteroids; OS oral corticosteroids; $\mathrm{Cl}$ : confidence interval. ${ }^{*}$ : in the first year of participating in the Second Dutch National Survey of General Practice (DNSGP-2). 


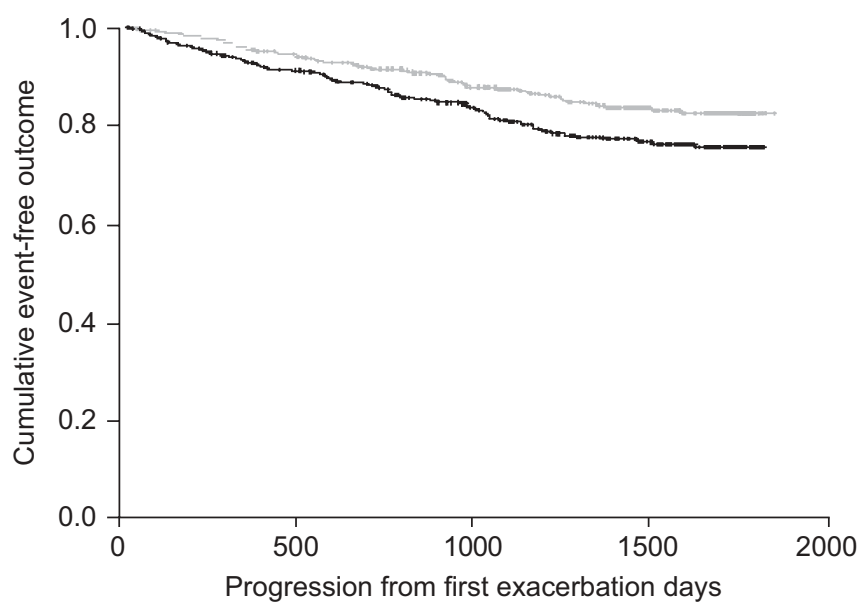

FIGURE 3. Kaplan-Meier estimates of the cumulative survival stratified according to treatment type (black line: oral corticosteroids only; grey line: oral corticosteroids and antibiotics). Difference between both treatment groups $p=0.02$, by log-rank. $n=842$

OS. Therefore, the present authors believe that bias suspected at this point, if present, would be associated with an underestimation of the effect of treatment.

An antibiotic course without oral corticosteroids seemed to work even better than OSA in preventing subsequent exacerbations. Unfortunately, it could not be derived from the database whether the antibiotic courses were prescribed for an exacerbation of COPD in all cases. Nevertheless, this finding is remarkable and appears to support the conclusion that antibiotics are beneficial in the prevention of exacerbations.

The exacerbation rate of the selected patients was 1.5 per patient per yr. This indicates that patients with more severe COPD were selected, as the exacerbation rate in general practice in the Netherlands is usually $<1$ per patient per yr [16]. Exacerbations in patients with more severe COPD are more likely to benefit from antibiotic treatment [2-4, 17]. The stronger treatment effect that was found in the time to third exacerbation compared with the time to second exacerbation suggests that the conclusions of the present study may be especially applicable to patients with recurrent exacerbations.

\section{Comparison with existing literature}

Most studies evaluate short-term outcomes: acute resolution rates and early relapse rates. It has previously been noted that use of the time to the next exacerbation as a primary outcome measure is especially suitable in evaluating COPD exacerbations [18-20]. As hypothesised, effective antibiotic treatment that results in bacterial eradication may prevent recurrence [18-20]. In one study, for the first five months after antibiotic treatment a significant difference was demonstrated between two antibiotics in the recurrence rate of exacerbations, relating to differences in induced bacterial eradication [21]. These effects of antibiotic treatment may become apparent in longterm evaluations, while short-term outcomes may not show such differences, as is the case in community-based studies of exacerbations comparing antibiotic and placebo [4, 22].
The present results extend, in a well-defined COPD population, the results of the current authors' previous study [5], in which patients were selected from the PHARMO database [6] on the basis of prescribed medication. The annual rate of exacerbations, treatment of first exacerbation (OS versus OSA), and the use of respiratory maintenance medication were comparable in both studies. A significant difference between both studies was the effect of treatment during the time between the first and second exacerbation, which was present in the previous study [5] but not the current study. In the current authors' previous study [5] featuring the PHARMO database [6], prescriptions by other prescribers were also included; however, selecting patients treated by GPs $(74 \%)$ resulted in smaller though still significant differences between treatment groups. This could be explained by the difference between pharmacy- and GP-based data, in that GPs' prescriptions are not always redeemed from the pharmacy, resulting in some patients with perhaps less severe symptoms not using the prescribed medication. This may account for $\sim 10 \%$ of prescriptions in the DNSGP-2/LINH study, resulting in a diminished treatment effect [23].

Bacteria and viruses are of influence in inducing exacerbations. Superadded bacterial infection after viral infection occurs frequently, and both bacteria and viruses may interact in a complex inflammatory process [24-27]. The beneficial effect of antibiotics described in the present study could be attributed to the fact that antibiotics may cause bacterial eradication. Airway inflammation is increased at the time of an exacerbation [28-30], as is systemic inflammation [31, 32]. In addition, in clinically stable, chronically Haemophilus influenzae-infected COPD patients, airway inflammation is more pronounced compared to that of non-infected patients [33]. Systemic inflammation contributes substantially to overall mortality in COPD patients [34, 35]. It can be hypothesised that the observed survival benefit of OSA in the present study is related to a stronger effect on systemic inflammation than treatment with OS. Recently, bacterial colonisation was found to be related to a higher frequency of exacerbations in patients with moderate-to-severe COPD, suggesting clinical relevance of the presence of bacteria in the lower airways [36]. The present study's finding of the beneficial role of antibiotics on the long-term recurrence rate is in line with these observations.

\section{Conclusion}

Until recently, studies have only shown a significant shortterm effect of antibiotic treatment in more severe patients and in hospitalised patients [1-4]. The present study now shows (long-term) benefits for patients treated by GPs. However, due to the retrospective character of the present study and its limitations with regard to the availability of characteristics of patients and exacerbations, the results presented cannot be seen as definitive proof of the long-term effects of antibiotics but should be confirmed in randomised clinical trials.

Even one exacerbation can have a large and sustained effect on health status. Although the initial recovery can be fast, the period to full recovery may be long [37]. A number of patients do not recover to baseline symptoms $[12,38]$ and a decline in forced expiratory volume in one second as a result of the exacerbation might contribute to this process $[39,40]$. Early reexacerbation is more common in patients with a deteriorated 
health status after an exacerbation and in patients with severe COPD; this delays the course of recovery [36]. Patients with frequent exacerbations have a lower quality of life, an increased risk of hospital admission and greater mortality, and generate more costs than patients with less frequent exacerbations. Postponing the next exacerbation can contribute to delaying this process of deterioration.

In the present study of primary care chronic obstructive pulmonary disease patients who used respiratory maintenance treatment, the addition of antibiotics to oral corticosteroids in the treatment of an exacerbation was associated with a reduced risk of a subsequent exacerbation; this was particularly the case in patients with recurrent exacerbations. Moreover, during follow-up, a survival benefit was demonstrated in the patients treated with antibiotics and corticosteroids. If confirmed in future prospective studies, these observations may have a major impact on exacerbation management in chronic obstructive pulmonary disease patients. Before general implementation, however, the pros and cons of antibiotic use, in particular the risk of increasing rates of resistance among respiratory pathogens, should be weighed against each other.

\section{ACKNOWLEDGEMENTS}

The present authors would like to thank R.B. Geskus (Academic Medical Centre, University of Amsterdam, Amsterdam, the Netherlands) for his support in the data analysis.

\section{REFERENCES}

1 Allegra L, Blasi F, de Bernardi B, Cosentini R, Tarsia P. Antibiotic treatment and baseline severity of disease in acute exacerbations of chronic bronchitis: a re-evaluation of previously published data of a placebo-controlled randomized study. Pulm Pharmacol Ther 2001; 14: 149-155.

2 Anthonisen NR, Manfreda J, Warren CP, Hershfiekd ES, Harding GK, Nelson NA. Antibiotic therapy in exacerbations of chronic obstructive pulmonary disease. Ann Intern Med 1987; 106: 196-204.

3 Saint S, Bent S, Vittinghoff E, Grady D. Antibiotics in chronic obstructive pulmonary disease exacerbations. A meta-analysis. JAMA 1995; 273: 957-960.

4 Ram FS, Rodriguez-Roisin R, Granados-Navarrete A, Garcia-Aymerich J, Barnes NC. Antibiotics for exacerbations of chronic obstructive pulmonary disease. Cochrane Database Syst Rev 2006; 2: CD004403.

5 Roede BM, Bresser P, Bindels PJE, et al. Antibiotic treatment is associated with reduced risk of a subsequent exacerbation in obstructive lung disease: a historical population-based cohort study. Thorax 2008; 63: 968-973.

6 PHARMO Institute. www.pharmo.nl. Date last accessed: 10 December, 2008.

7 Westert GP, Schellevis FG, de Bakker DH, Groenewegen JM, Bensing JM, van der Zee J. Monitoring health inequalities through general practice: the Second National Survey of General Practice. Eur J of Public Health 2005; 15: 59-65.

8 Verheij RA, te Brake JHM, Abrahamse H, van den Hoogen $\mathrm{H}$, Braspenning $\mathrm{J}$, van Althuis T. Landelijk Informatienetwerk Huisartsenzorg. Feiten en cijfers over huisartsenzorg in Nederland. Utrecht/Nijmegen: nivel wok. www.LINH.nl (Netherlands Information Network of General Practice). Date last accessed: 10 December, 2008.

9 WONCA (World Organization of Family Doctors Classification Committee). ICPC-2-R: International Classification of Primary care. Revised 2nd Edn. Singapore, WONCA, 2005.

10 Lau HS, de BA, Beuning KS, Porsius A. Validation of pharmacy records in drug exposure assessment. J Clin Epidemiol 1997; 50: 619-625.

11 Roede BM, Bindels PJ, Brouwer HJ, Bresser $\mathrm{P}$, de Borgie CA, Prins JM. Antibiotics and steroids for exacerbations of COPD in primary care: compliance with Dutch guidelines. Br J Gen Pract 2006; 56: 662-665.

12 Seemungal TA, Donaldson GC, Bhowmik A, Jeffries DJ, Wedzicha JA. Time course and recovery of exacerbations in patients with chronic obstructive pulmonary disease. Am J Respir Crit Care Med 2000; 161: 1608-1613.

13 Kelly PJ, Lim LL-Y. Survival analysis for recurrent event data: an application to childhood infectious diseases. Statist Med 2000; 19: 13-33.

14 Therneau TM, Grambsch PM. Modeling Survival Data: Extending the Cox Model. New York, Springer-Verlag, 2000; pp. 231-260.

15 Niewoehner DE, Lokhnygina Y, Rice K, et al. Risk indexes for exacerbations and hospitalizations due to COPD. Chest 2007; 131: 20-28.

16 Schermer TR, Saris CG, van den Bosch WJ, et al. Exacerbations and associated healthcare cost in patients with COPD in general practice. Monaldi Arch Chest Dis 2006; 65: 133-140.

17 Woodhead M, Blasi F, Ewig S, et al. Guidelines for the management of adult lower respiratory tract infections. Eur Respir J 2005; 26: 1138-1180.

18 Miravitlles M, Torres A. Antibiotics in exacerbations of COPD: lessons from the past. Eur Respir J 2004; 24: 896-897.

19 Chodosh S. Clinical significance of the infection-free interval in the management of acute bacterial exacerbations of chronic bronchitis. Chest 2005; 127: 2231-2236.

20 Miravitlles M. Exacerbations of chronic obstructive pulmonary disease: when are bacteria important? Eur Respir J 2002; 20: Suppl. 36, 9s-19s.

21 Wilson R, Allegra L, Huchon G, et al. Short-term and longterm outcomes of moxifloxacin compared to standard antibiotic treatment in acute exacerbations of chronic bronchitis. Chest 2004; 125: 953-964.

22 Sachs AP, Koëter GH, Groenier KH, van der Waaij D, Schiphuis J, Meyboom-deJong B. Changes in symptoms, peak expiratory flow, and sputum flora during treatment with antibiotics of exacerbations in patients with chronic obstructive pulmonary disease in general practice. Thorax 1995; 50: 758-763.

23 Hagström B, Mattsson B, Rost IM, Gunnarsson RK. What happened to the prescriptions? A single, short, standardized telephone call may increase compliance. Family Practice 2004; 21: 46-50.

24 Wilkinson TM, Hurst JR, Perera WR, Wilks M, Donaldson GC, Wedzicha JA. Effect of interactions between lower airway bacterial and rhinoviral infection in exacerbations of COPD. Chest 2006; 129: 317-324. 
25 Sajjan US, Jia Y, Newcomb DC, et al. H. influenzae potentiates airway epithelial cell responses to rhinovirus by increasing ICAM-1 and TLR3 expression. FASEB J 2006; 20: 2121-2123.

26 Roger T, Bresser P, Snoek M, et al. Exaggerated IL-8 and IL-6 responses to TNF-alpha by parainfluenza virus type 4infected NCI-H292 cells. Am J Physiol Lung Cell Mol Physiol 2004; 287: L1048-L1055.

27 Dev D, Wallace E, Sankaran R, et al. Value of C-reactive protein measurements in exacerbations of chronic obstructive pulmonary disease. Respir Med 1998; 92: 664-667.

28 Wedzicha JA, Seemungal TA, MacCallum PK, et al. Acute exacerbations of chronic obstructive pulmonary disease are accompanied by elevations of plasma fibrinogen and serum IL-6 levels. Thromb Haemost 2000; 84: 210-215.

29 Schols AM, Buurman WA, Staal van den Brekel AJ, Dentener MA, Wouters EF. Evidence for a relation between metabolic derangements and increased levels of inflammatory mediators in a subgroup of patients with chronic obstructive pulmonary disease. Thorax 1996; 51: 819-824.

30 Walter RE, Wilk JB, Larson MG, et al. Systemic inflammation and COPD: the Framingham Heart Study. Chest 2008; 133: $19-25$.

31 Groenewegen KH, Postma DS, Hop WC, et al. Increased systemic inflammation is a risk factor for COPD exacerbations. Chest 2008; 133: 350-357.

32 Bresser P, Out TA, van Alphen L, Jansen KM, Lutter R. Airway inflammation in nonobstructive and obstructive chronic bronchitis with chronic Haemophilus influenzae airway infection. Comparison with noninfected patients with chronic obstructive pulmonary disease. Am J Respir Crit Care Med 2000; 162: 947-952.

33 Dahl M, Vestbo J, Lange P, Bojesen SE, Tybjaerg-Hansen A, Nordestgaard BG. C-reactive protein as a predictor of prognosis in chronic obstructive pulmonary disease. Am J Respir Crit Care Med 2007; 175: 250-255.

34 Man SF, Connett JE, Anthonisen NR, Wise RA, Tashkin DP, Sin DD. C-reactive protein and mortality in mild to moderate chronic obstructive pulmonary disease. Thorax 2006; 61: 849-853.

35 Patel IS, Seemungal TA, Wilks M, Lloyd-Owen SJ, Donaldson GC, Wedzicha JA. Relationship between bacterial colonisation and the frequency, character, and severity of COPD exacerbations. Thorax 2002; 57: 759-764.

36 Spencer S, Jones PW. Time course of recovery of health status following an infective exacerbation of chronic bronchitis. Thorax 2003; 58: 589-593.

37 Donaldson GC, Wedzicha JA. COPD exacerbations. 1: Epidemiology. Thorax 2006; 61: 164-168.

38 Kanner RE, Anthonisen NR, Connett JE. Lower respiratory illnesses promote FEV1 decline in current smokers but not ex-smokers with mild chronic obstructive pulmonary disease: results from the Lung Health Study. Am J Respir Crit Care Med 2001; 164: 358-364.

39 Donaldson GC, Seemungal TA, Bhowmik A, Wedzicha JA. Relationship between exacerbation frequency and lung function decline in chronic obstructive pulmonary disease. Thorax 2002; 57: 847-852.

40 Spencer S, Calverley PM, Burge PS, Jones PW. Impact of preventing exacerbations on deterioration of health status in COPD. Eur Respir J 2004; 23: 698-702. 